\title{
Infrared regularization with spin-3/2 fields
}

\author{
Véronique Bernard ${ }^{\dagger} \# 1$, Thomas R. Hemmert*\#2, Ulf-G. Meißner ${ }^{\ddagger \# 3}$ \\ †Université Louis Pasteur, Laboratoire de Physique Théorique \\ F-67084 Strasbourg, France \\ * Technische Universität München, Physik Department T-39 \\ D-85747 Garching, Germany \\ $¥$ Universität Bonn, Helmholtz Institut für Strahlen- und Kernphysik (Theorie) \\ Nußallee 14-16, D-53115 Bonn, Germany.
}

\begin{abstract}
We present a Lorentz-invariant formulation of baryon chiral perturbation theory including spin-3/2 fields. Particular attention is paid to the projection on the spin-3/2 components of the delta fields. We also discuss the nucleon mass and the pion-nucleon sigma term.
\end{abstract}

\#1 email: bernard@lpt6.u-strasbg.fr

\#2 email: themmert@physik.tu-muenchen.de

\#3 email: meissner@itkp.uni-bonn.de 
1. The delta resonance plays a special role in low-energy nuclear and particle physics, due to its near mass degeneracy with the nucleon and its strong couplings to pions, nucleons and photons. It was therefore argued early that spin-3/2 (decuplet) states should be included in baryon chiral perturbation theory [1, which is the low-energy effective field theory of the Standard Model. The work of [1] and subsequent authors made use of the heavy baryon approach, which treats the baryons as static sources like in heavy quark effective field theory and allows for a systematic power counting in the presence of matter fields, as pioneered in [2] and systematically explored in 3]. Also, special care has to be taken about the decoupling of resonances in the chiral limit [4. This approach was systematized by counting the nucleon-delta mass splitting as an additional small parameter in Ref. [5], the corresponding power counting was called the "small scale expansion". The heavy baryon approach has been successfully applied to a variety of processes, for reviews see [6, 7], and a status report on chiral effective field theories with deltas is given in [8]. More recently, a Lorentz-invariant formulation of baryon chiral perturbation theory has become available [9], the so-called "infrared regularization" (IR). A similar approach had been presented earlier in [10. Such a formulation allows to include strictures from analyticity and is thus particularly suited for extensions of chiral perturbation theory based on dispersion relations, see e.g. 11, 12, 13, 14, 15, 16, 17. Furthermore, the use of the Dirac propagator as opposed to the static fermion propagator in the heavy baryon scheme allows for a resummation of important recoil effects, see e.g. the work on electromagnetic form factors [18] or the nucleon spin structure [19, 20]. The chiral expansion of the baryon masses also seems to converge faster in this scheme 21] whereas the description of pion-nucleon scattering is not yet in a satisfactory status [22, 23. The renormalization of relativistic baryon chiral perturbation theory has been discussed in detail in [24]. In this Letter, we give a consistent extension of the infrared regularization method in the presence of spin-3/2 \#4 . What is new here is that we explicitly project onto the spin-3/2 parts and that the method easily allows to include any external source in a chiral and gauge invariant fashion (in contrast to the recent proposal in [25]). As simple applications, we calculate the delta loop contribution to the nucleon self-energy and the pion-nucleon sigma term.

2. In this section, we briefly review the formalism necessary to describe spin- $3 / 2$ fields. More details can e.g. be found in [26, 27, 28]. We first write down the propagator for a free spin-3/2 field (called the delta propagator from here on) in $d$ space-time dimensions,

$$
\mathcal{G}_{\mu \nu}^{\Delta}(p)=-\frac{\not p+m_{\Delta}}{p^{2}-m_{\Delta}^{2}}\left\{g_{\mu \nu}-\frac{1}{d-1} \gamma_{\mu} \gamma_{\nu}-\frac{(d-2) p_{\mu} p_{\nu}}{(d-1) m_{\Delta}^{2}}+\frac{p_{\mu} \gamma_{\nu}-p_{\nu} \gamma_{\mu}}{(d-1) m_{\Delta}}\right\},
$$

with $m_{\Delta}$ the delta mass. This may be rewritten by projection onto the spin $3 / 2$ and $1 / 2$ components

$$
\mathcal{G}_{\mu \nu}^{\Delta}(p)=-\frac{\not p+m_{\Delta}}{p^{2}-m_{\Delta}^{2}} P_{\mu \nu}^{3 / 2}-\frac{1}{\sqrt{d-1} m_{\Delta}}\left(\left(P_{12}^{1 / 2}\right)_{\mu \nu}+\left(P_{21}^{1 / 2}\right)_{\mu \nu}\right)+\frac{d-2}{(d-1) m_{\Delta}^{2}}\left(\not p+m_{\Delta}\right)\left(P_{22}^{1 / 2}\right)_{\mu \nu}
$$

with

$$
\begin{aligned}
P_{\mu \nu}^{3 / 2} & =g_{\mu \nu}-\frac{1}{d-1} \gamma_{\mu} \gamma_{\nu}-\frac{1}{(d-1) p^{2}}\left(\not p \gamma_{\mu} p_{\nu}+p_{\mu} \gamma_{\nu} \not p\right)-\frac{d-4}{d-1} \frac{p_{\mu} p_{\nu}}{p^{2}} \\
\left(P_{12}^{1 / 2}\right)_{\mu \nu} & =\frac{1}{\sqrt{d-1} p^{2}}\left(p_{\mu} p_{\nu}-\not p p_{\nu} \gamma_{\mu}\right) \\
\left(P_{21}^{1 / 2}\right)_{\mu \nu} & =\frac{1}{\sqrt{d-1} p^{2}}\left(\not p p_{\mu} \gamma_{\nu}-p_{\mu} p_{\nu}\right) \\
\left(P_{22}^{1 / 2}\right)_{\mu \nu} & =\frac{p_{\mu} p_{\nu}}{p^{2}}
\end{aligned}
$$

These spin projection operators fulfill the orthogonality relations

$$
\left(P_{i j}^{I}\right)_{\mu \nu}\left(P_{k l}^{J}\right)^{\nu \rho}=\delta_{I J} \delta_{j k}\left(P_{i l}^{I}\right)_{\mu}^{\rho}, \quad(I, J)=1,2, \quad(i, j, k, l)=1,2 .
$$

Note the infrared singular pieces $\sim 1 / p^{2}$ appearing in the spin-projected parts of the propagator, which will play an important role later on. Also, we remark that the spin- $1 / 2$ pieces do not propagate and thus one should

\footnotetext{
\#4 The inclusion of the delta when one separates loop integrals into soft and hard parts was already considered in [23].
} 
be able to absorb their contribution in purely polynomial terms (which amounts to a redefinition of certain low-energy constants in the effective field theory). If one considers processes where the center-of-mass energy reaches the delta mass, $\sqrt{s}=m_{\Delta}$, the free delta propagator has to be resummed as described in detail e.g. in [10, 25]. Let us now briefly discuss the leading chiral pion-delta-nucleon Lagrangian. It is given by

$$
\mathcal{L}_{\pi \Delta N}=c_{A} \bar{\Psi}_{\mu}^{i} \Theta_{\nu}^{\mu}(Z) w_{i}^{\nu} \psi+\text { h.c. }
$$

where $\Psi_{\mu}$ describes the Rarita-Schwinger (spin-3/2) field, $\psi$ the nucleon doublet, $w_{i}^{\mu}=\operatorname{Tr}\left(\tau_{i} u^{\mu}\right) / 2$ the axial current and $\Theta_{\mu \nu}=g_{\mu \nu}+(Z-1 / 2) \gamma_{\mu} \gamma_{\nu}$. The dependence on the so-called off-shell parameter $Z$ is, however, spurious, since it does not appear in the spin-3/2 contributions and thus physical observables do not depend on it. The axial-vector coupling constant $c_{A}$, which is related to the pion-delta-nucleon coupling $g_{\pi \Delta N}$, is frequently called $h_{A}$ in the literature. More precisely, what appears in Eq. (15) is the the coupling $c_{A}$ in the chiral limit. For simplicity, we use in the following the SU(4) coupling constant relation

$$
c_{A}=h_{A}=\frac{3}{2 \sqrt{2}} g_{A} .
$$

The resulting value is somewhat larger than found in typical fits say to pion-nucleon scattering data or from fitting the $\Delta \rightarrow N \pi$ width, see e.g. 29, 30. We remark that like in Eq.(5), we are using standard chiral-invariant couplings of the $\pi \Delta N$ system, because differences to other couplings appearing in the literature can simply be absorbed in the polynomial contributions to be discussed in the following.

3. Next, we make some short remarks about the effective field theory of massive spin- $1 / 2$ fields chirally coupled to Goldstone bosons and external sources, called baryon chiral perturbation theory. It is complicated by the fact that the nucleon mass does not vanish in the chiral limit and thus introduces a new mass scale apart from the ones set by the quark masses. Therefore, any power of the quark masses can be generated by chiral loops in the nucleon (baryon) case, spoiling the one-to-one correspondence between the loop expansion and the one in the small parameter $q$. One method to overcome this is the heavy mass expansion (called heavy baryon chiral perturbation theory, for short HBCHPT) where the nucleon mass is transformed from the propagator into a string of vertices with increasing powers of $1 / \mathrm{m}$. Then, a consistent power counting emerges $\# 5$. However, this method has the disadvantage that certain types of diagrams are at odds with strictures from analyticity. The best example is the so-called triangle graph, which enters e.g. the scalar form factor or the isovector electromagnetic form factors of the nucleon. In a fully relativistic treatment, such constraints from analyticity are automatically fulfilled. It was argued in 10 that relativistic one-loop integrals can be separated into "soft" and "hard" parts. While for the former the power counting as in HBCHPT applies, the contributions from the latter can be absorbed in certain LECs. In this way, one can combine the advantages of both methods. A more formal and rigorous implementation of such a program was given in [9. The underlying method is called "infrared regularization". Any dimensionally regularized one-loop integral $H$ is split into an infrared singular (called $I$ ) and a regular part (called $R$ ) by a particular choice of Feynman parameterization,

$$
H=I+R .
$$

Consider first the regular part. If one chirally expands the contributions to $R$, one generates polynomials in momenta and quark masses. Consequently, to any order, $R$ can be absorbed in the LECs of the effective Lagrangian. On the other hand, the infrared (IR) singular part has the same analytical properties as the full integral $H$ in the low-energy region and its chiral expansion leads to the non-trivial momentum and quark-mass dependences of CHPT, like e.g. the chiral $\operatorname{logs}$ or fractional powers of the quark masses. For a typical oneloop integral (like e.g. the nucleon self-energy $\Sigma$ ) this splitting can be achieved in the following way (omitting prefactors)

$$
\begin{aligned}
\Sigma=\int \frac{d^{d} k}{(2 \pi)^{d}} \frac{1}{A B} & =\int_{0}^{1} d z \int \frac{d^{d} k}{(2 \pi)^{d}} \frac{1}{[(1-z) A+z B]^{2}} \\
& =\left\{\int_{0}^{\infty}-\int_{1}^{\infty}\right\} d z \int \frac{d^{d} k}{(2 \pi)^{d}} \frac{1}{[(1-z) A+z B]^{2}}=I+R
\end{aligned}
$$

\footnotetext{
${ }^{\# 5}$ The extension of this method for including deltas treating the $N \Delta$ mass splitting as an additional small parameter has been given in [5].
} 
with $A=M_{\pi}^{2}-k^{2}-i \epsilon, B=m^{2}-(p-k)^{2}-i \epsilon, \epsilon \rightarrow 0^{+}, M_{\pi}$ the pion mass and $d$ the number of space-time dimensions. Any general one-loop diagram with arbitrary many insertions from external sources can be brought into this form by combining the propagators to a single pion and a single nucleon propagator. It was also shown that this procedure leads to a unique, i.e. process-independent result, in accordance with the chiral Ward identities of QCD [9]. Consequently, the transition from any one-loop graph $H$ to its IR singular piece $I$ defines a symmetry-preserving regularization. For more details, the reader is referred to $\underline{9}$.

4. Next, we develop a systematic infrared regularization for effective field theories with spin-3/2 fields. This should be considered the main result of this paper. Due to the presence of the IR singular terms $\sim 1 / p^{2}$ when one projects the delta propagator onto its spin-3/2 and spin- $1 / 2$ components, cf. Eq.(3), one has to deal with a type of integrals that do not appear in the pure pion-nucleon approach. To be specific, consider the integral appearing in the self-energy (details of the self-energy calculation will be given below)

$$
\begin{aligned}
J_{0}^{0}\left(p^{2}\right) & =\frac{1}{i} \int \frac{d^{4} k}{(2 \pi)^{4}} \frac{-1}{(k-p)^{2}\left(M_{\pi}^{2}-k^{2}\right)} \\
& =\frac{1}{(2 \pi)^{4}} \int_{0}^{1} d x \int d^{4} k \frac{1}{\left[k^{2}+p^{2} x(x-1)+M_{\pi}^{2}(1-x)\right]^{2}} \\
& =\frac{\pi^{d}}{(2 \pi)^{d}} \frac{\Gamma(2-d / 2)}{\Gamma(2)} \int_{0}^{1} d x \frac{1}{(1-x)^{2-d / 2}\left(-p^{2} x+M_{\pi}^{2}\right)^{2-d / 2}} .
\end{aligned}
$$

In the region of low momenta, where the chiral expansion is expected to converge quickly, the first factor in the denominator is of $\mathcal{O}\left(q^{0}\right)$, while the second is of $\mathcal{O}\left(q^{2}\right)$ so that the chiral dimension of the integral should be $\mathcal{O}\left(q^{d-2}\right)$. We remark that at $x=1$, the integrand develops a pole. Following [9], we perform a change of variables

$$
x=\frac{M_{\pi}^{2}}{p^{2}} u \equiv \alpha u
$$

so that

$$
J_{0}^{0}\left(p^{2}\right)=\frac{\pi^{d / 2}}{(2 \pi)^{d}} \frac{\Gamma(2-d / 2)}{\Gamma(2)} \frac{1}{p^{2}} \int_{0}^{1 / \alpha} d u \frac{1}{(1-\alpha u)^{2-d / 2}(1-u)^{2-d / 2}}\left(M_{\pi}^{2}\right)^{d / 2-1} .
$$

We see that a complication arises due to the infrared singular piece $\sim 1 /(k-p)^{2}$ in the spin-projected parts of the propagator. Specifically, due to the term $\sim \alpha u^{2}$, the denominator brings some additional $\alpha$ contribution when performing the integral. $J_{0}^{0}\left(p^{2}\right)$ turns out to behave as $M_{\pi}^{d-4}$ instead of the expected $M_{\pi}^{d-2}$. If one evaluates this integral straightforwardly, one finds that it contains a logarithmic contribution that indeed has the correct chiral behavior. The disturbing contribution is due to the factor $(1-\alpha u)$ which stems from the pole at $x=1$ of the infrared singular part of the propagator. In fact, such contributions should be contained in the regular part, since they have nothing to do with the chiral expansion. To formally achieve this separation, one rewrites the integral as $(d=4-2 \varepsilon)$

$$
\begin{aligned}
\int_{0}^{1 / \alpha} d u \frac{1}{(1-\alpha u)^{2-d / 2}(1-u)^{2-d / 2}} & =\int_{0}^{1 / \alpha} d u(1-\varepsilon \ln (1-\alpha u)-\varepsilon \ln (1-u)) \\
& =\int_{0}^{1 / \alpha} d u\left((1-\varepsilon \ln (1-\alpha u)-1)+\int_{0}^{1 / \alpha} d u \frac{1}{(1-u)^{2-d / 2}} .\right.
\end{aligned}
$$

From the second integral one then obtains the irregular part by letting $1 / \alpha \rightarrow \infty$. Therefore, the separation of the integral $J_{0}^{0}\left(p^{2}\right)$ into the irregular and the regular terms reads, $J_{0}^{0}\left(p^{2}\right)=I_{0}^{0}\left(p^{2}\right)+R_{0}^{0}\left(p^{2}\right)$,

$$
\begin{aligned}
I_{0}^{0}\left(p^{2}\right) & =\frac{\pi^{d / 2}}{(2 \pi)^{d}} \frac{\Gamma(2-d / 2)}{\Gamma(2)} \frac{1}{p^{2}} \int_{0}^{\infty} d u \frac{1}{(1-u)^{2-d / 2}}\left(M_{\pi}^{2}\right)^{d / 2-1}, \\
R_{0}^{0}\left(p^{2}\right) & =\frac{\pi^{d / 2}}{(2 \pi)^{d}} \frac{\Gamma(2-d / 2)}{\Gamma(2)}\left\{\int_{0}^{1} d x\left[\frac{1}{(1-x))^{2-d / 2}}-1\right]-\int_{1}^{\infty} d x \frac{1}{\left(-p^{2} x+M_{\pi}^{2}\right)^{2-d / 2}}\right\} .
\end{aligned}
$$

The irregular part can now be worked out using the methodology developed in [9], we find

$$
I_{0}^{0}\left(p^{2}\right)=-\frac{M_{\pi}^{2}}{p^{2}}\left[2 \bar{\lambda}+\frac{1}{16 \pi^{2}} \ln \frac{M_{\pi}^{2}}{m^{2}}\right]=-\frac{\Delta_{\pi}}{p^{2}} .
$$




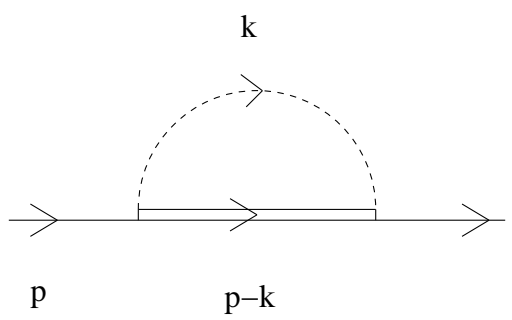

Figure 1: Feynman diagram for the nucleon self-energy with an spin-3/2 intermediate state. Solid, double and dashed lines denote nucleons, deltas and pions, in order.

Here, the piece $\sim \bar{\lambda}$ contains a pole term,

$$
\bar{\lambda}=\frac{m^{d-4}}{16 \pi^{2}}\left\{\frac{1}{d-4}-\frac{1}{2}\left(\ln 4 \pi+\gamma_{E}+1\right)\right\}
$$

with $\gamma_{E}$ the Euler-Mascheroni constant and we have set the running scale of dimensional regularization, $\lambda$, to be the nucleon mass [9]. Furthermore, the pion tadpole contribution $\Delta_{\pi}$ is given by the well-known loop integral

$$
\Delta_{\pi}=\frac{1}{i} \int \frac{d^{d} k}{(2 \pi)^{2}} \frac{1}{M_{\pi}^{2}-k^{2}}
$$

Of course, the scale that naturally appears in loop integrals with deltas is $m_{\Delta}$, not $m$, as used in Eqs. (15 16). However, the differences due to this choice of scale can be absorbed in polynomial (regular) terms, so that one can work with one scale only in the coupled pion-nucleon-delta system. However, to assess the theoretical uncertainty at a given order, one can set $\lambda=m_{\Delta}$ in the delta loop diagrams. Eq. (15) also shows that the non-propagating parts of the projected propagator will be absorbed in tadpole contributions, as it is expected since such contributions can be understood as a very heavy delta shrunk to a point-like vertex.

Armed with this prescription, we can now set up a consistent expansion in small external momenta, pion masses and the $N \Delta$ mass splitting $\Delta=m_{\Delta}-m$, in complete analogy to the small scale expansion formulated for heavy spin-3/2 fields [5. We therefore refrain from discussing here in any detail the corresponding power counting and the construction of the chiral effective Lagrangian (most of this is available in the literature). As discussed in detail in 31, special care has to be taken to fulfill the decoupling theorem of QCD, which states that leading chiral singularities can not be modified by resonance fields. From here on, all small parameters (quark masses, external momenta, nucleon-delta mass splitting) are collectively denoted as $\epsilon$.

5. We first calculate the leading one-loop contribution to the nucleon self-energy with an intermediate delta without any reference to a special regularization and with the full propagator, Eq.(1), see Fig. 11 This is done for the following reasons. First, such a calculation allows one to study different regularization procedures. Second, one can explicitly study the role of the non-propagating spin-1/2 components. Third, one can also easier compare with existing calculations in the literature. We have

$$
\Sigma_{\Delta}\left(p^{2}\right)=-\frac{9}{4} \frac{g_{A}^{2}}{F_{\pi}^{2}} i \int \frac{d^{4} k}{(2 \pi)^{4}}\left[k^{\mu}-\left(Z+\frac{1}{2}\right) \not k \gamma^{\mu}\right] \mathcal{G}_{\mu \nu}^{\Delta}(p-k) \frac{1}{M_{\pi}^{2}-k^{2}}\left[k^{\nu}-\left(Z+\frac{1}{2}\right) \gamma^{\nu} \not \not\right],
$$

The general structure of this integral is

$$
\Sigma_{\Delta}\left(p^{2}\right)=\mathrm{C} \frac{1}{i} \int \frac{d^{4} k}{(2 \pi)^{4}}(A(k)+B(k) \not p+C(k) \not k)
$$

with $\mathrm{C}=9 g_{A}^{2} /\left(4 F_{\pi}^{2}\right)$, and the functions $A(k), B(k)$ and $C(k)$ can be straightforwardly evaluated. After integration, the self-energy can be written as

$$
\Sigma_{\Delta}\left(p^{2}\right)=\tilde{A}+\not p \tilde{B}=\tilde{A}+\tilde{B} m+(\not p-m) \tilde{B},
$$


where the functions $\tilde{A}$ and $\tilde{B}$ follow after integration of Eq.(19). The nucleon mass shift due to the delta intermediate state is (setting $Z=-1 / 2$ for simplicity)

$$
\begin{aligned}
\delta m= & \left.(\tilde{A}+\tilde{B} m)\right|_{p^{2}=m^{2}} \\
= & \frac{(d-2) \mathrm{C}}{(d-1) m_{\Delta}^{2}}\left(-\frac{1}{2 d} m_{\Delta}^{2} m \Delta_{\Delta}-\frac{1}{2} m_{\Delta} m^{2} \Delta_{\Delta}+\frac{\Delta}{4}\left(m_{\Delta}+m\right) m \Delta_{\Delta}+\frac{\Delta}{2}\left(m_{\Delta}+m\right)^{2} m^{2} J_{1}\left(m^{2}\right)\right. \\
& \quad+M_{\pi}^{2} m^{2}\left(m_{\Delta}+m\right) J_{0}\left(m^{2}\right)-\frac{M_{\pi}^{2}}{4} \Delta_{\Delta}-m M_{\pi}^{2} J_{1}\left(m^{2}\right)\left[m^{2}+\frac{1}{2} m_{\Delta} m+\frac{1}{2} m_{\Delta}^{2}\right] \\
& \left.\quad+\frac{1}{4} M_{\pi}^{4} m J_{1}\left(m^{2}\right)+M_{\pi}^{2} \Delta_{\pi}\left[m_{\Delta}+m+\frac{1}{2 d} m\right]\right),
\end{aligned}
$$

in terms of the loop functions

$$
\begin{aligned}
J_{0}\left(p^{2}\right) & =\frac{1}{i} \int \frac{d^{d} k}{(2 \pi)^{2}} \frac{1}{\left(M_{\pi}^{2}-k^{2}\right)\left(m_{\Delta}^{2}-(p-k)^{2}\right)}, \\
p_{\mu} J_{1}\left(p^{2}\right) & =\frac{1}{i} \int \frac{d^{d} k}{(2 \pi)^{2}} \frac{k_{\mu}}{\left(M_{\pi}^{2}-k^{2}\right)\left(m_{\Delta}^{2}-(p-k)^{2}\right)}, \\
\Delta_{\Delta} & =\frac{1}{i} \int \frac{d^{d} k}{(2 \pi)^{2}} \frac{1}{m_{\Delta}^{2}-k^{2}},
\end{aligned}
$$

and we have defined

$$
\Delta \equiv m_{\Delta}-m \text {. }
$$

In IR, we have $\Delta_{\Delta}=0$, and $J_{0}\left(m^{2}\right)\left(J_{1}\left(m^{2}\right)\right)$ is of order $\mathcal{O}(\epsilon)\left(\mathcal{O}\left(\epsilon^{2}\right)\right)$, so that to lowest (third) order

$$
\begin{aligned}
\delta m & =\frac{(d-2) \mathrm{C}}{(d-1) m_{\Delta}^{2}}\left[\frac{1}{2} \Delta\left(m+m_{\Delta}\right)^{2} m^{2} J_{1}\left(m^{2}\right)+M_{\pi}^{2} m^{2}\left(m+m_{\Delta}\right) J_{0}\left(m^{2}\right)\right] \\
& =-\frac{3 g_{A}^{2}}{16 \pi^{2} F_{\pi}^{2}}\left[\Delta\left(\Delta^{2}-\frac{3}{2} M_{\pi}^{2}\right) \ln \frac{M_{\pi}^{2}}{m^{2}}+2\left(\Delta^{2}-M_{\pi}^{2}\right)^{3 / 2} \ln \left(\frac{\Delta}{M_{\pi}}+\sqrt{\frac{\Delta^{2}}{M_{\pi}^{2}}-1}\right)-\frac{\Delta}{2}\left(\frac{4}{3} \Delta^{2}-M_{\pi}^{2}\right)\right],
\end{aligned}
$$

where the last equation is valid for $\Delta^{2}>M_{\pi}^{2}$ (as it is the case in nature). This result agrees with the one in 30. Note also that for a more compact notation and easier comparison with the existing literature, we have retained some higher order terms in the formula expressing the mass shift in terms of the loop functions $J_{0}$ and $J_{1}$.

As noted above, we have not yet separated the spin- $3 / 2$ from the spin- $1 / 2$ components. Since the spin- $1 / 2$ components do not propagate, their contribution should be entirely absorbed in polynomial pieces (contact terms). Utilizing the projection operators given in Eq.(31), the spin-3/2 contribution to the self-energy takes the form

$$
\Sigma_{\Delta}^{3 / 2}\left(p^{2}\right)=-\frac{(d-2) \mathrm{C}}{d-1} \int \frac{d^{4} k}{(2 \pi)^{4}}\left(k^{2} p^{2}-(k \cdot p)^{2}\right) \frac{-\left(\not p+m_{\Delta}-\not p\right)}{(k-p)^{2}\left(M_{\pi}^{2}-k^{2}\right)\left(m_{\Delta}^{2}-(k-p)^{2}\right)},
$$

which leads to the nucleon mass-shift (we give here only the result employing IR)

$$
\begin{gathered}
\delta m^{3 / 2}=\frac{(d-2) \mathrm{C}}{(d-1) m_{\Delta}^{2}}\left[M_{\pi}^{2} m^{2}\left(J_{0}\left(m^{2}\right)\left(m_{\Delta}+m\right)-m J_{1}\left(m^{2}\right)\right)+\frac{1}{2} m^{2}\left(\Delta\left(m_{\Delta}+m\right)-M_{\pi}^{2}\right)\left(m_{\Delta}+m\right) J_{1}\left(m^{2}\right)\right. \\
+\frac{1}{4}\left(-\Delta\left(m_{\Delta}+m\right)+M_{\pi}^{2}\right)^{2} m J_{1}\left(m^{2}\right)-M_{\pi}^{2} m^{2}\left(J_{0}^{0}\left(m^{2}\right)\left(m_{\Delta}+m\right)-J_{1}^{0}\left(m^{2}\right) m\right) \\
\left.+\frac{m^{2}}{2}\left(M_{\pi}^{2}+m^{2}\right)\left(m_{\Delta}+m\right) J_{1}^{0}\left(m^{2}\right)-\frac{m}{4}\left(M_{\pi}^{2}+m^{2}\right)^{2} J_{1}^{0}\left(m^{2}\right)\right]
\end{gathered}
$$

in terms of the new loop functions $J_{0}^{0}\left(p^{2}\right)$ defined in Eq.(9) and

$$
p_{\mu} J_{1}^{0}\left(p^{2}\right)=\frac{1}{i} \int \frac{d^{d} k}{(2 \pi)^{2}} \frac{-k_{\mu}}{(p-k)^{2}\left(M_{\pi}^{2}-k^{2}\right)} .
$$


We also have the loop function relation $J_{1}^{0}\left(p^{2}\right)=\left(p^{2}+M_{\pi}^{2}\right) J_{0}^{0}\left(p^{2}\right) /\left(2 p^{2}\right)+\Delta_{\pi} /\left(2 p^{2}\right)$ (in dimensional regularization). Noting now that the loop functions $J_{0}^{0}\left(m^{2}\right)$ and $J_{1}^{0}\left(m^{2}\right)$ are of order $\mathcal{O}\left(\epsilon^{2}\right)$ in the chiral expansion, the lowest (third) order result for the mass shift derived from Eq.(26) agrees with the one given in Eq.(24) but differs of course in the higher order terms. It is also important to stress that the spin- $3 / 2$ contribution to the mass shift is independent of $Z$, as it should be.

Next, we consider the spin- $1 / 2$ contribution to the self-energy, $\Sigma_{\Delta}^{1 / 2}\left(p^{2}\right)$. The corresponding mass shift has the form (we again give for simplicity the result for $Z=-1 / 2$ )

$$
\begin{aligned}
\delta m^{1 / 2}=\frac{3 g_{A}^{2}}{2 F_{\pi}^{2} m_{\Delta}^{2}} \frac{1}{256} M_{\pi}^{4}[ & 2 \ln \frac{M_{\pi}^{2}}{m^{2}}\left(2\left(m_{\Delta}+m\right)+\frac{2 M_{\pi}^{2}}{m^{2}}\left(2 m+m_{\Delta}\right)-\frac{M_{\pi}^{4}}{m^{3}}\right) \\
& \left.+\frac{1}{3}\left(\left(m+4 m_{\Delta}\right)+4 \frac{M_{\pi}^{2}}{m^{2}}\left(2 m+m_{\Delta}\right)-2 \frac{M_{\pi}^{4}}{m^{3}}\right)\right] .
\end{aligned}
$$

We have thus shown explicitly that these contributions can be completely absorbed into polynomial terms appearing in the chiral expansion of the nucleon mass beyond leading one-loop order 32, 9,

$$
m_{N}=m_{0}-4 c_{1} M_{\pi}^{2}-\frac{3 g_{A}^{2} M_{\pi}^{3}}{32 \pi F_{\pi}^{2}}+k_{1} M_{\pi}^{4} \ln \frac{M_{\pi}}{m}+k_{2} M_{\pi}^{4}+\mathcal{O}\left(M_{\pi}^{5}\right),
$$

where $c_{1}, k_{1}$ and $k_{2}$ are (combinations of) dimension two and four low-energy constants and $m_{0}$ is the nucleon mass in the chiral limit. Furthermore, all $Z$-dependence is of course included in these polynomial terms, too.

From the mass shift, one can directly derive the delta loop contribution to the sigma term using the FeynmanHellmann theorem,

$$
\sigma_{\pi N}^{\Delta}(0)=M_{\pi}^{2} \frac{d \delta m}{d M_{\pi}^{2}}
$$

To leading order one has (using the full or the spin $3 / 2$ part of the propagator):

$$
\begin{aligned}
\sigma_{\pi N}^{\Delta}(0) & =\frac{M_{\pi}^{2}\left(m+m_{\Delta}\right)}{m_{\Delta}^{2}} \mathrm{C}\left(m^{2} J_{0}\left(m^{2}\right)+\frac{1}{6} \frac{1}{4 \pi^{2}} \frac{m^{2}+2 m_{\Delta}^{2}}{m^{2}} \Delta\left(m+m_{\Delta}\right)\right) \\
& =\frac{\mathrm{C} M_{\pi}^{2}}{4 \pi^{2}}\left[\Delta \ln \frac{M_{\pi}}{m}+\sqrt{\Delta^{2}-M_{\pi}^{2}} \ln \left(\frac{\Delta}{M_{\pi}}+\sqrt{\frac{\Delta^{2}}{M_{\pi}^{2}}-1}\right)\right] .
\end{aligned}
$$

Eq. (31) naturally agrees with the result of Ref. [23] but differs by a polynomial contribution in $\Delta$ from the one of [10] due to the use of a $d$-independent propagator in that paper. Again, for easier comparison we have retained some higher order terms in the upper expression in Eq. (31). This concludes the formalism and we now turn to a numerical evaluation of the mass shift and the sigma term.

6. We are now in the position to evaluate the delta loop contribution to the nucleon mass shift and the sigma term. We use $g_{A}=1.267, M_{\pi}=139.57 \mathrm{MeV}, F_{\pi}=92.4 \mathrm{MeV}$ and $\Delta=271 \mathrm{MeV}$. From Eq.(6) it follows that $c_{A}=1.34$. We remark that the resulting numbers scale linearly with the axial-vector coupling constant $c_{A}$. The delta contribution to the nucleon mass shift and the sigma term are collected in Table 1 We give the lowest (third) order result which is the same if one uses the full or the projected spin-3/2 propagator, as pointed out earlier. However, in IR the integrals also contain higher order pieces which can be retained (see e.g. the detailed discussion in [18]). In that case, one has to project onto the spin- $3 / 2$ pieces to get rid of the unphysical contribution from the spin- $1 / 2$ components. However, as shown in the table, the difference is numerically irrelevant for the observables considered here. Furthermore, these higher order corrections amount to a very small correction to the lowest order result. This, however, is not always the case, see e.g. [18. We also remark a certain sensitivity to the scale of dimensional regularization. This dependence, however, will be balanced by counter terms not considered here. The results shown in Table 1 are not very different from earlier ones obtained in the heavy baryon approach [34, 33, which is expected since to this order neither the delta loop contribution to the nucleon mass shift nor to the sigma term is very sensitive to recoil corrections or similar effects. We stress again that a complete analysis of the nucleon mass or the sigma term would require a novel determination of certain low-energy constants to fulfill the requirements of decoupling. This, however, goes beyond the scope of this Letter. 


\begin{tabular}{|l|cc|}
\hline & $\delta m[\mathrm{MeV}]$ & $\sigma_{\pi N}^{\Delta}(0)[\mathrm{MeV}]$ \\
\hline lowest order & $86.4(108.1)$ & $-45.6(-60.0)$ \\
unexpanded, with full propagator & $87.2(112.7)$ & $-46.3(-63.3)$ \\
unexpanded, with spin-3/2 propagator & $88.1(113.7)$ & $-44.7(-61.4)$ \\
\hline
\end{tabular}

Table 1: Delta contribution to the nucleon mass shift and the sigma term. Lowest order refers to the third order IR result based on Eq. 24). Unexpanded means that the higher order terms from the various loop functions are retained. The propagator used is also given (in the lowest order case, using the full or the spin-3/2 part of the propagator leads to the same result, as discussed in the text). The numbers in the round brackets are obtained with $\lambda=m_{\Delta}$.

7. In this Letter, we constructed a systematic infrared regularization for chiral effective field theories including spin-3/2 fields. To arrive at a consistent formulation, one has to project onto the spin- $3 / 2$ components of these fields. This projection leads to a new type of integrals as compared to the pure pion-nucleon theory. We have shown that the IR scheme of [9] can be extended systematically and furthermore, this procedure allows for a chiral invariant inclusion of external sources, like e.g. photons or weak currents. The contributions from the spin-1/2 components get completely absorbed into the polynomial pieces of the effective Lagrangian. Furthermore, the frequently used off-shell parameters $(X, Y, Z)$ are also contained in these polynoms and thus do not lead to observable consequences. It is now important to apply this method to observables, where $\Delta \pi$ loops are expected to play a significant role, like in all types of Compton scattering. Work along these lines is underway.

TRH thanks the Université Louis Pasteur for hospitality, where part of this work was done.

\section{References}

[1] E. Jenkins and A. V. Manohar, Phys. Lett. B 259 (1991) 353.

[2] E. Jenkins and A. V. Manohar, Phys. Lett. B 255 (1991) 558.

[3] V. Bernard, N. Kaiser, J. Kambor and U.-G. Meißner, Nucl. Phys. B 388 (1992) 315.

[4] J. Gasser and A. Zepeda, Nucl. Phys. B 174 (1980) 445.

[5] T. R. Hemmert, B. R. Holstein and J. Kambor, J. Phys. G 24 (1998) 1831 arXiv:hep-ph/9712496.

[6] V. Bernard, N. Kaiser and U.-G. Meißner, Int. J. Mod. Phys. E 4 (1995) 193 arXiv:hep-ph/9501384.

[7] U.-G. Meißner, in Shifman, M. (ed.): "At the frontier of particle physics", vol. 1, pp. 417-505 (World Scientific, Singapore, 2001) arXiv:hep-ph/0007092.

[8] T. R. Hemmert, arXiv:nucl-th/0105051

[9] T. Becher and H. Leutwyler, Eur. Phys. J. C 9 (1999) 643 arXiv:hep-ph/9901384.

[10] P. J. Ellis and H. B. Tang, Phys. Rev. C 57 (1998) 3356 arXiv:hep-ph/9709354.

[11] T. N. Truong, CERN-TH-4748/87.

[12] J. F. Donoghue, J. Gasser and H. Leutwyler, Nucl. Phys. B 343 (1990) 341.

[13] J. Gasser and U.-G. Meißner, Nucl. Phys. B 357 (1991) 90.

[14] J. A. Oller and E. Oset, Phys. Rev. D 60 (1999) 074023 arXiv:hep-ph/9809337.

[15] U.-G. Meißner and J. A. Oller, Nucl. Phys. A 673 (2000) 311 arXiv:nucl-th/9912026.

[16] B. Ananthanarayan and P. Büttiker, Eur. Phys. J. C 19 (2001) 517 arXiv:hep-ph/0012023.

[17] B. Ananthanarayan, G. Colangelo, J. Gasser and H. Leutwyler, Phys. Rept. 353 (2001) 207 arXiv:hep-ph/0005297.

[18] B. Kubis and U.-G. Meißner, Nucl. Phys. A 679 (2001) 698. arXiv:hep-ph/0007056.

[19] V. Bernard, T. R. Hemmert and U.-G. Meißner, Phys. Lett. B 545 (2002) 105 arXiv:hep-ph/0203167.

[20] V. Bernard, T. R. Hemmert and U.-G. Meißner, arXiv:hep-ph/0212033 Phys. Rev. D 67 (2003), in press.

[21] P. J. Ellis and K. Torikoshi, Phys. Rev. C 61 (2000) 015205 arXiv:nucl-th/9904017.

[22] T. Becher and H. Leutwyler, JHEP 0106 (2001) 017 arXiv:hep-ph/0103263.

[23] K. Torikoshi and P. J. Ellis, arXiv:nucl-th/0208049 
[24] T. Fuchs, J. Gegelia, G. Japaridze and S. Scherer, arXiv:hep-ph/0302117

[25] V. Pascalutsa and D. R. Phillips, arXiv:nucl-th/0212024

[26] W. Rarita and J. S. Schwinger, Phys. Rev. 60 (1941) 61.

[27] M. Benmerrouche, R. M. Davidson and N. C. Mukhopadhyay, Phys. Rev. C 39 (1989) 2339.

[28] V. Pascalutsa and R. Timmermans, Phys. Rev. C 60 (1999) 042201 arXiv:nucl-th/9905065.

[29] N. Fettes and U.-G. Meißner, Nucl. Phys. A 679 (2001) 629 arXiv:hep-ph/0006299.

[30] T. R. Hemmert, B. R. Holstein, J. Kambor and G. Knöchlein, Phys. Rev. D 57 (1998) 5746 arXiv:nucl-th/9709063.

[31] V. Bernard, H. W. Fearing, T. R. Hemmert and U.-G. Meißner, Nucl. Phys. A 635 (1998) 121 [Erratumibid. A 642 (1998) 563] arXiv:hep-ph/9801297.

[32] B. Borasoy and U.-G. Meißner, Annals Phys. 254 (1997) 192 arXiv:hep-ph/9607432 .

[33] V. Bernard, N. Kaiser and U.-G. Meißner, Z. Phys. C 60 (1993) 111 arXiv:hep-ph/9303311.

[34] E. Jenkins, Nucl. Phys. B 368 (1992) 190. 